\title{
Integrating endobronchial ultrasound bronchoscopy with molecular testing of immunotherapy biomarkers in non-small cell lung cancer
}

\author{
Steven Bozinovski ${ }^{1}$, Amanda Vannitamby ${ }^{1}$, Kanishka Rangamuwa ${ }^{2}$, Savreet Aujla ${ }^{1}$, Hao Wang ${ }^{1}$, Christian Aloe ${ }^{1}$, \\ Louis Irving ${ }^{2}$, Tracy T. Leong ${ }^{3,4,5}$, Daniel P. Steinfort ${ }^{2,5}$ \\ ${ }^{1}$ School of Health \& Biomedical Sciences, RMIT University, Bundoora, Victoria, Australia; ${ }^{2}$ Department of Respiratory Medicine, Royal Melbourne \\ Hospital, Melbourne, Australia; ${ }^{3}$ Department of Respiratory Medicine, Austin Health, Heidelberg, Victoria, Australia; ${ }^{4}$ The Walter and Eliza Hall \\ Institute of Medical Research, Parkville, Victoria, Australia; ${ }^{5}$ Faculty of Medicine, University of Melbourne, Parkville, Victoria, Australia \\ Contributions: (I) Conception and design: All authors; (II) Administrative support: S Bozinovski, A Vannitamby, DP Steinfort, TT Leong, C Aloe, H \\ Wang; (III) Provision of study materials or patients: None; (IV) Collection and assembly of data: A Vannitamby, DP Steinfort, C Aloe, H Wang, S \\ Bozinovski; (V) Data analysis and interpretation: None; (VI) Manuscript writing: All authors; (VII) Final approval of manuscript: All authors. \\ Correspondence to: Prof. Steven Bozinovski. School of Health \& Biomedical Sciences, RMIT University, Bundoora, VIC 3083, Australia. \\ Email: steven.bozinovski@rmit.edu.au.
}

\begin{abstract}
Immunotherapy has transformed treatment of advanced non-small-cell lung cancer (NSCLC) patients leading to remarkable long-term survival benefit. However, only about $20 \%$ of advanced NSCLC patients typically respond to immune checkpoint inhibitors (ICIs) that target the PD-1/PD-L1 pathway. The only validated biomarker for ICI therapy is the PD-L1 immunohistochemistry (IHC) test, which is considered an imperfect assay due to several variables including availability and integrity of tumour tissue, variability in staining/scoring techniques and heterogeneity in PD-L1 protein expression within and across tumour biopsies. Herein, we discuss integrating minimally invasive EBUS bronchoscopy procedures with novel molecular approaches to improve accuracy and sensitivity of PD-L1 testing. EBUS guided bronchoscopy facilitates repeated sampling of tumour tissue to increase the probability of detecting PD-L1 positive tumours. Since intra-tumoural PD-L1 (CD274) copy number is reported to be less heterogeneous than PD-L1 protein detection, quantifying PD-L1 transcript levels may increase detection of PD-L1 positive tumours. PD-L1 transcript levels show excellent concordance with PD-L1 IHC scoring and multiplex digital droplet PCR (ddPCR) assays that quantify absolute $P D-L 1$ transcript copy number have been developed. ddPCR can also be automated for high throughput detection of low abundant variants with excellent sensitivity and accuracy to improve the broader application of diagnostic cut-off values. Optimizing diagnostic workflows that integrate optimal EBUS bronchoscopy procedures with emerging molecular ICI biomarker assays may improve the selection criteria for ICI therapy benefit.
\end{abstract}

Keywords: Immunotherapy; biomarkers; programmed death Inhibitor 1 ligand (PD-L1); lung cancer; non-small cell lung cancer (NSCLC); bronchoscopy

Submitted Jun 26, 2020. Accepted for publication Aug 19, 2020.

doi: $10.21037 /$ tlcr-20-781

View this article at: http://dx.doi.org/10.21037/tlcr-20-781 


\section{Introduction}

Immune checkpoint inhibitors (ICIs) are currently changing how advanced non-small cell lung cancer (NSCLC) patients that do not harbour actionable mutations are being treated. Programmed death inhibitor 1 (PD-1) and cytotoxic T-lymphocyte-associated protein 4 (CTLA-4) are classic inhibitory immune checkpoint receptors that are critical in maintaining self-tolerance, as they will transmit inhibitory signals to prevent tissue pathology when engaged by their ligands. Tumour cells can exploit this process and evade cytotoxic $\mathrm{T}$ cell immuno-surveillance by expressing the PD-1 ligand (PD-L1). Engagement of PD-1 expressing tumour-infiltrating CD8+ T cells with PD-L1 on tumour cells suppresses cytotoxic $\mathrm{T}$ cell cytokine production and proliferation in NSCLC (1). In the past five years, multiple checkpoint inhibitors targeting PD-1, PD-L1 and/ or CTLA-4 have been approved and targeted for use in treating advanced, refractory, or relapsed solid tumours that have not responded to standard-of-care cancer treatments. Nivolumab is a fully human IgG4 anti-PD-1 antibody that improves overall survival compared to docetaxel monotherapy in non-squamous NSCLC patients (2) and response rates were even better in squamous cell carcinoma (SCC) patients (3). Pembrolizumab is an alternative humanized monoclonal antibody against $\mathrm{PD}-1$, which significantly improved overall survival in NSCLC patients compared to platinum-based chemotherapy (4).

\section{Limitations of established and emerging immunotherapy biomarkers}

About $20 \%$ of advanced NSCLC patients typically respond to biologics that target the $\mathrm{PD}-1 / \mathrm{PD}-\mathrm{L} 1$ pathway and this response can be doubled by screening for PD-L1 tumours levels (4), however the majority of advanced NSCSLC patients will not respond to ICI monotherapy. The assessment of PD-L1 tumour staining by immunohistochemistry (IHC) is currently the only validated predictive biomarker for ICI therapy in NSCLC (5). Pembrolizumab is now approved as a first line therapy for NSCLC patients with $\geq 50 \%$ PD-L1 tumour expression and a second line therapy for $\geq 1 \%$ PD-L1 tumour expression (6). The rationale for selecting ICI responders with predictive biomarkers extend beyond enriching for response or resistance. Toxicities including underlying autoimmune disorders represent a typical exclusion criterion in ICI therapy trials, as reactivation of primed lymphocytic populations can be detrimental or fatal. Biologics such as monoclonal antibodies that suppress the PD-1/PDL1 pathway are also very expensive, thereby limiting the broad application of this therapy. Hence, the relatively low response rate and potential toxicities relating to ICI therapy necessitate the need to identify predictive biomarkers to guide treatment.

PD-L1 IHC is established as the principal ICI response biomarker in NSCLC, as discussed above. However, a number of limitations of PD-L1 IHC have been recognized in both the clinic and the published literature where the evaluation of PD-L1 expression in advanced NSCLC patients can be technically challenging for pathologists and oncologists. Studies validating PD-L1 tumour expression included only histologic specimens, however in clinical practice diagnostic specimens are more frequently cytologic/small volume specimens. Hence, the availability and quality of fixed tissue for PD-L1 testing is variable in practice, as small bronchoscopy specimens can be crushed and unsuitable for IHC. In addition, the process of collecting, processing and staining tissue blocks is not necessarily standardised across different sites and this can collectively influence test performance and accuracy. Direct comparison between different PD-L1 IHC assays suggests that individual tests cannot be interchangeably used in clinical practice (7), however other groups have shown that 22C3, 28-8, SP263 but not SP142 can be used interchangeably (8).

In addition to the technical challenges of scoring PDL1 using small bronchoscopy specimens, heterogeneity of PD-L1 expression remains an unresolved question and variability according to metastatic site is recognized $(9,10)$. Temporal heterogeneity has been reported following adjuvant therapy $(10,11)$, emphasizing the importance of re-biopsy in patients who experience recurrent/progressive disease. Intra-patient heterogeneity has been reported following multi-site biopsy $(9,12)$ with sufficient variance to result in alteration in clinical management. Heterogeneity may be attributable in part to variation in NSCLC histotype (13), and presents significant implications for its accuracy as a predictive biomarker. Use of more advanced molecular assessment, such as copy number alterations, may improve concordance in PD-L1 assessment compared to IHC (14), but this remains to be confirmed. Hence, PDL1 IHC is considered an imperfect test, where there are NSCLC patients that are negative for PD-L1 expression, but still respond to ICI therapy. Nonetheless, screening PD-L1 levels still represents an important strategy for 
selecting NSCLC patients for ICI therapy, but there are opportunities to improve the workflow and evaluation of PD-L1 expression to minimise technical challenges and subjective interpretation of results.

Tumour mutational burden (TMB) is emerging as an independent biomarker for ICI benefit. It is broadly defined as the total number of somatic mutations in the tumour exome (15) and is associated with neoantigen burden which can activate $\mathrm{T}$ lymphocytes to kill cancer cells (16). In the context of clinical trials, a high TMB has been shown to have greater predictive capacity than PD-L1 IHC in advanced NSCLC (17). This concept was validated in the CheckMate 227 study, the first randomised phase III trial evaluating ICI outcome in NSCLC patients predefined in relation to TMB. In this open label trial, the combination of nivolumab plus ipilimumab (anti-CTLA-4) was particularly effective in increasing one year progression-free survival in patients with a high TMB (at least 10 mutations per megabase) relative to chemotherapy, irrespective of PDL1 expression level (17). Furthermore, determining TMB has become increasingly feasible with the availability of sequencing technology. However, challenges arise with regards to tumour tissue quantity and quality; lack of standardisation in TMB assessment methods; and variability in definition of high TMB. Efforts are being made to harmonise TMB distributions across different cohorts (18), and this may help to determine TMB threshold and validate the clinical value of TMB as a predictive biomarker. Recent clinical studies have also defined tumour immunophenotypes that correlate with response to ICI (19). Inflamed ("hot") tumours contain a high density of infiltrating cytotoxic CD3+ lymphocytes with an "inflamed" gene expression profile (20), providing a more favourable environment for T-cell activation and expansion. "Hot" tumours, where the suppressive phenotype is driven by upregulation of immune checkpoints such as PD-1 and CTLA-4, demonstrate a high rate of response to ICIs. Non-inflamed ("cold") tumours associated with immune suppression or tolerance (19) exhibit lower rates of response to ICIs.

\section{Bronchoscopic sampling may address some limitations of PD-L1 IHC testing}

Heterogeneity of PD-L1 expression within and across tumour specimens from an individual creates significant challenges in utilising the PD-L1 IHC test as an accurate and predictive biomarker. To address this, extensive intra- tumoural and inter-tumoral sampling may increase the probability of detecting PD-L1 positive tumour cells. Endobronchial ultrasound (EBUS) sampling of tumours routinely involves multiple samples (21) taken from multiple sites (22), which provides a greater breadth of assessment to potentially overcome intra-lesional and inter-lesional heterogeneity. EBUS guided bronchoscopy is a minimally invasive technique that facilitates repeated sampling of tumour tissue. EBUS guided sampling when combined with rapid-on-site examination (ROSE) can diagnose malignancy in real time with high sensitivity and specificity (23-26). Specimens can be processed as a cell block for morphologic and immunohistochemical assessment (27). While initial studies regarding the predictive value of PD-L1 were limited to histologic specimens, recent studies have confirmed that, with adequate tumour cellularity, PD-L1 IHC on cytology cell-block specimens (including EBUS-directed specimens) is an acceptable alternative to histological specimens in NSCLC (28). Sampling of pulmonary parenchymal lesions is undertaken using radial EBUS, a different method which includes both cytologic and histologic sampling. Cytologic samples may be assessed by IHC (29), as well as nextgeneration sequencing (including kRas) (23).

Bronchoscopic cryobiopsy can achieve larger parenchymal lung specimens than possible through standard EBUS-forceps biopsy (30). Hence, radial EBUSdirected sampling may be augmented by use of cryobiopsy $(31,32)$ and our group has used this for assessment of tumour-infiltrating lymphocyte density (unpublished data). In addition, EBUS-guided transbronchial needle aspiration (EBUS-TBNA) is used for diagnosis of mediastinal/ hilar lymphadenopathy (25). While initially designed for diagnostic sampling of pathologic lymphadenopathy, EBUS-TBNA is increasingly used for systematic staging of normal-size lymph nodes $(22,33)$. Samples yield significant amounts of highly intact DNA (34), and our group has used fresh tissue acquired by EBUS-TBNA to complete whole genome sequencing to map genomic heterogeneity in inoperable lung cancer (35). From this dataset we have been able to establish TMB scores (unpublished data). In addition, EBUS-TBNA sampling has recently been used to complete immunophenotypic characterization of tumourdraining lymph nodes in NSCLC (36).

\section{Molecular analysis of PD-L1 levels may reduce heterogeneity in testing results}

Elevated PD-L1 levels in the tumour microenvironment 
can be driven by amplification of the PD-L1 (CD274) gene in certain cases. A study comparing PD-L1 IHC scores with $P D-L 1$ gene amplification in pulmonary carcinoma patients detected $P D-L 1$ gene amplification in about $9 \%$ cases and $12 / 14$ of the amplified cases demonstrating higher PD-L1 IHC scores (37). Furthermore, whilst $P D$ L1 copy number was moderately concordant with PDL1 IHC scores in EBUS-TBNA biopsies, intra-tumoural $P D-L 1$ copy number was less heterogeneous than PDL1 IHC staining in resected tumour tissue (14). There are also other biological processes within the tumour microenvironment that will drive an increase in PD-L1 levels, which will be discussed later. Since they all converge of increasing $P D-L 1$ transcript levels, there are biological and practical reasons for redirecting PD-L1 testing towards a molecular approach. The comparison of $P D-L 1$ transcript levels with PD-L1 IHC tumour cell scoring has shown excellent agreement in NSCLC biopsies, and supports the quantitative determination of $P D-L 1$ transcript levels as an alternative molecular approach that minimises interobserver variability $(38,39)$. We have also developed a novel approach to detect PD-L1 status and multiple tumour mutations using a single EBUS bronchoscopy specimen. In this study, $P D-L 1$ transcript expression determined by RT-qPCR was highly concordant with PD-L1 IHC tumour cell staining in NSCLC, as determined using the Ventana (SP263) PDL1 Assay (40). In contrast to tumour staining, there was a weaker association between $P D-L 1$ transcript levels and PD-L1 immune cell staining (40).

The question remains whether molecular approaches can be adapted to the clinical diagnostic setting and improve the application of predictive biomarkers for ICI therapies. The rapid advancement of molecular technologies is providing an unparalleled opportunity to transform the clinical diagnostics field in lung cancer. It is increasingly feasible to isolate tumour DNA and screen for mutations from primary and metastatic sites. The sensitivity and accuracy of molecular technology is also facilitating the detection of low abundance circulating tumour DNA in blood to screen for genetic mutations in a non-invasive and dynamic manner in advanced patients. An example of such technology is digital droplet PCR (ddPCR), which is now established for plasma genotyping of venous blood samples. This procedure allows for the rapid detection of common EGFR mutations including the EGFR p.T790M acquired resistance mutation in patients with multiple metastatic site (41). ddPCR is particularly adept at detecting low abundant variants and reported to be more sensitive than amplification-refractory mutation system (ARMS) for detection of EGFR variants, and may be more useful in monitoring disease progression (42). In addition, ddPCR can address some limitations associated with RT-qPCR as it allows for absolute quantification of mRNA copy number to generate diagnostic cut-off values and is more suitable for higher order multiplexing of gene targets.

We have developed a multiplex ddPCR assay where absolute MMP9, TIMP3 and PD-L1 transcript copy numbers were determined within a single assay using Taqman primers and the QX200 Droplet Digital PCR System (43). The MMP9:TIMP3 ratio was significantly elevated in NSCLC biopsies and was highly sensitive (99\%) for discriminating malignant biopsies. Analysis of the PDL1:TIMP3 ratio in the same assay revealed that $P D-L 1$ transcript levels are significantly increased in biopsies with $\geq 50 \%$ PD-L1 IHC tumour cell score $(\mathrm{P}<0.0001)(43)$. Hence, our novel ddPCR approach can accurately quantify PD-L1 tumour levels and provide sufficient nucleic acid for additional genetic tests including EGFR mutations or ALK and ROS1 fusions from a single small biopsy, thereby potentially avoiding the need for re-biopsy. ddPCR technology also has the capacity to be automated, performed within 24 hours of specimen collection and quantitative analysis removes potential inter-assay and inter-observer variation in the scoring of PD-L1 levels. Whilst our findings found that PD-L1 tumour expression is the dominant driver of increased PD-L1 transcript levels in NSCLC biopsies, our approach does not differentiate between tumour and immune cell PD-L1 expression. What is becoming increasingly apparent is that PD-L1 expression on tumour and infiltrating immune cells has a non-redundant role in regulating immunity to cancer, where immune cell expression is equally important in predicting benefit to atezolizumab in NSCLC (44). Future studies need to evaluate the utility of our ddPCR test as a predictive independent biomarker, as the assessment of clinical response is the 'gold standard' to compare different ICI therapy biomarker strategies.

\section{Analysis of molecular regulators that control PD-L1 expression}

An alternative approach to developing ICI biomarkers may be to quantify the molecular regulators that control expression of inhibitory receptor ligands such as PDL1. We now understand that the increase in PD-L1 expression in solid tumours can be regulated by multiple 
mechanisms. $P D-L 1$ gene expression can be controlled in an interferon-gamma-inducible manner, whereby IRF1 transcription factor binds to the CD274/PD-L1 promoter region via a JAK/STAT dependent mechanism (45). The inflammatory nature of the solid tumour microenvironment includes activated innate and adaptive immune cells that can produce interferons, which can control the spatial and temporal expression of PD-L1 in tumour and immune cells. Retrospective analysis of interferon regulated genes in advanced melanoma patients developed a 28 -gene signature score, which showed a significant correlation with overall response and progression-free survival to ICI therapy (46). Whilst the importance of interferons in regulating PD-L1 in NSCLC requires further investigation, it may not be a uniform mechanism as the association between ICI response and interferon gene signatures appears to be weaker in NSCLC when compared to melanoma (47).

Another plausible mechanism by which PD-L1 expression is increased in NSCLC is the oncogene-driven upregulation of $P D-L 1$ gene expression at a transcriptional level. kRas is a commonly mutated oncogene in western countries that is present in about $30 \%$ of adenocarcinomas, where it is more commonly detected in smokers. kRas has been considered undruggable due to its high affinity for GTP and lack of clear binding pocket, however new generation inhibitors have now been developed and are showing more rapid and durable tumour regression in mice (48). The RAF/MEK/ERK MAPK signalling pathway is the classic kRas effector pathway that controls cellular proliferation, differentiation and survival. Activation of the MAPK kinase pathway also converges on the c-Jun and STAT3 transcription factors, which can increase expression of PD-L1 transcript levels (49). In addition, increased oncogenic kRas signalling can stabilize PD-L1 mRNA, which drives tumour cell-intrinsic PD-L1 expression and immunoresistance (50).

There is emerging data to suggest that kRas status can influence survival outcomes in response to ICI therapies. As an independent biomarker, kRas status does not appear to influence nivolumab efficacy, where patients were treated irrespective of PD-L1 expression (51). However, in a separate study that factored in PD-L1 expression, the efficacy of ICIs was consistently higher in $\mathrm{kRas}{ }^{\text {mutant }} \mathrm{PD}-\mathrm{L} 1^{\text {high }}$ patients compared to $\mathrm{kRas}{ }^{\text {wildtype }} \mathrm{PD}-\mathrm{L} 1^{\text {high }}$ patients (52). In a recent meta-analysis of five trials involving over 3000 patients with advanced NSCLC, patients receiving ICIs displayed prolonged overall survival in the kRas mutant subgroup but not in the kRas wild-type subgroup compared to docetaxel (53). A similar finding was observed in a smaller meta-analysis of three trials where ICIs significantly improved overall survival in previously treated $\mathrm{kRas}^{\text {mutant }}$ patients, but not in kRas ${ }^{\text {wildype }}$ patients (54).

Activating EGFR mutations can also stimulate PDL1 expression in tumour and immune cells (55). However, unlike kRas mutant tumours, harboring an activating EGFR mutation does not appear to confer improved response to ICI therapy in NSCLC (56). This likely reflects the observation that EGFR wild-type tumours are more likely to express higher PD-L1 than mutated tumours (57). In contrast to EGFR mutations, the presence of ALK fusions were positively associated with increased PD-L1 expression (57). NSCLC cell lines that are positive for ALK-fusions also display increased expression of PD-L1 levels that was dependent on MAPK and AKT signaling and sensitive to the ALK inhibitor alectinib (58). Gene deletion of Phosphatase and tensin homolog (PTEN) tumour suppressor commonly occurs in NSCLC and is associated with more aggressive tumours due to activation of the AKT/mTOR signaling pathways in cancer cells (59-63) . The genetic loss and inactivation of PTEN and Lbk1 in mice recapitulated pathological features of SCC and resulted in a marked increase in PD-L1 tumour expression (64). Furthermore, inhibition of the mTOR pathway effectively reduced PD-L1 levels in lung tumors from A/J mice exposed to the tobacco-carcinogen $\mathrm{NNK}$ and in $\mathrm{kRas}^{\mathrm{LA} 2}$ mice (64).

\section{Conclusions}

The screening of PD-L1 levels by IHC is relatively straightforward and cost-effective in the experienced diagnostic pathology setting, but it is not without its limitations. The availability of good quality tumour tissue, non-standardised tissue collection, processing and staining protocols and intra-tumoural heterogeneity collectively contribute to variability in PD-L1 IHC biomarker performance. Nonetheless, PD-L1 expression analysis remains the only validated approach to guiding ICI therapies. Since PD-L1 transcript levels show excellent concordance with PD-L1 tumour levels determined by IHC, there is an opportunity to track PD-L1 levels using new molecular technology that is highly sensitive and readily automated. Multiplex ddPCR assays can accurately quantify PD-L1 absolute copy number in EBUS bronchoscopy specimens (43). The integration of advanced tumour sampling procedures such as radial EBUS-directed 
sampling by cryobiopsy with ddPCR technology may offer an unparalleled level of sensitivity and accuracy to generate reliable prognostic cut-off values for PD-L1 expression. In summary, a diagnostic workflow that integrates the collection of designated molecular biospecimens by EBUS bronchoscopy will facilitate evaluation of multiple ICI molecular biomarkers including ddPCR based PD-L1, TMB or kRas status. The sensitivity of such techniques now makes it feasible to simultaneously screen for multiple molecular biomarkers within a single EBUS sample, whereby a signature of ICI response combining two or more markers may prove to be optimal. This approach requires further clinical validation and can be integrated in the multitude of immunotherapy trials. Further studies are needed to validate and benchmark molecular ICI biomarkers in a coordinated approach, which ultimately strive to improve selection criteria for immunotherapy.

\section{Acknowledgments}

Funding: The study was supported by funding from NHMRC Australia.

\section{Footnote}

Provenance and Peer Review: This article was commissioned by the Guest Editor (Daniel Steinfort) for the series "Lung cancer and the immune system" published in Translational Lung Cancer Research. The article was sent for external peer review organized by the Guest Editor and the editorial office.

Peer Review File: Available at http://dx.doi.org/10.21037/ tlcr-20-781

Conflicts of Interest: All authors have completed the ICMJE uniform disclosure form (available at http://dx.doi. org/10.21037/tlcr-20-781). The series "Lung cancer and the immune system" was commissioned by the editorial office without any funding or sponsorship. DPS served as the unpaid Guest Editor of the series and serves as an unpaid editorial board member of Translational Lung Cancer Research from Sep 2019 to Sep 2021. The authors have no other conflicts of interest to declare.

Ethical Statement: The authors are accountable for all aspects of the work in ensuring that questions related to the accuracy or integrity of any part of the work are appropriately investigated and resolved.

Open Access Statement: This is an Open Access article distributed in accordance with the Creative Commons Attribution-NonCommercial-NoDerivs 4.0 International License (CC BY-NC-ND 4.0), which permits the noncommercial replication and distribution of the article with the strict proviso that no changes or edits are made and the original work is properly cited (including links to both the formal publication through the relevant DOI and the license). See: https://creativecommons.org/licenses/by-nc-nd/4.0/.

\section{References}

1. Zhang Y, Huang S, Gong D, et al. Programmed death-1 upregulation is correlated with dysfunction of tumorinfiltrating CD8+ T lymphocytes in human non-small cell lung cancer. Cell Mol Immunol 2010;7:389-95.

2. Borghaei H, Paz-Ares L, Horn L, et al. Nivolumab versus Docetaxel in Advanced Nonsquamous Non-Small-Cell Lung Cancer. N Engl J Med 2015;373:1627-39.

3. Brahmer J, Reckamp KL, Baas P, et al. Nivolumab versus Docetaxel in Advanced Squamous-Cell Non-Small-Cell Lung Cancer. N Engl J Med 2015;373:123-35.

4. Garon EB, Rizvi NA, Hui R, et al. Pembrolizumab for the treatment of non-small-cell lung cancer. N Engl J Med 2015;372:2018-28.

5. Leighl NB, Hellmann MD, Hui R, et al. Pembrolizumab in patients with advanced non-small-cell lung cancer (KEYNOTE-001): 3-year results from an openlabel, phase 1 study. The Lancet Respiratory Medicine 2019;7:347-57.

6. Pai-Scherf L, Blumenthal GM, Li H, et al. FDA Approval Summary: Pembrolizumab for Treatment of Metastatic Non-Small Cell Lung Cancer: First-Line Therapy and Beyond. Oncologist 2017;22:1392-9.

7. Hendry S, Byrne DJ, Wright GM, et al. Comparison of Four PD-L1 Immunohistochemical Assays in Lung Cancer. J Thorac Oncol 2018;13:367-76.

8. Luo L, Luo X, Chen W, et al. Consistency Analysis of Programmed Death-Ligand 1 Expression between Primary and Metastatic Non-Small Cell Lung Cancer: A Retrospective Study. J Cancer 2020;11:974-82.

9. Haragan A, Field JK, Davies MPA, et al. Heterogeneity of PD-L1 expression in non-small cell lung cancer: Implications for specimen sampling in predicting treatment response. Lung Cancer 2019;134:79-84.

10. Hong L, Negrao MV, Dibaj SS, et al. Programmed Death- 
Ligand 1 Heterogeneity and Its Impact on Benefit From Immune Checkpoint Inhibitors in NSCLC. J Thorac Oncol 2020;15:1449-59.

11. Lacour M, Hiltbrunner S, Lee SY, et al. Adjuvant Chemotherapy Increases Programmed Death-Ligand 1 (PD-L1) Expression in Non-small Cell Lung Cancer Recurrence. Clin Lung Cancer 2019;20:391-6.

12. Saito $\mathrm{Y}$, Horiuchi S, Morooka H, et al. Inter-tumor heterogeneity of PD-L1 expression in non-small cell lung cancer. J Thorac Dis 2019;11:4982-91.

13. Naso JR, Wang G, Pender A, et al. Intratumoral heterogeneity in programmed death-ligand 1 immunoreactivity is associated with variation in nonsmall cell lung carcinoma histotype. Histopathology 2020;76:394-403.

14. Yoshimura K, Inoue Y, Karayama M, et al. Heterogeneity analysis of PD-L1 expression and copy number status in EBUS-TBNA biopsy specimens of non-small cell lung cancer: Comparative assessment of primary and metastatic sites. Lung Cancer 2019;134:202-9.

15. Yarchoan M, Hopkins A, Jaffee EM. Tumor Mutational Burden and Response Rate to PD-1 Inhibition. N Engl J Med 2017;377:2500-1.

16. Havel JJ, Chowell D, Chan TA. The evolving landscape of biomarkers for checkpoint inhibitor immunotherapy. Nat Rev Cancer 2019;19:133-50.

17. Hellmann MD, Ciuleanu TE, Pluzanski A, et al. Nivolumab plus Ipilimumab in Lung Cancer with a High Tumor Mutational Burden. N Engl J Med 2018;378:2093-104.

18. Vokes NI, Liu D, Ricciuti B, et al. Harmonization of Tumor Mutational Burden Quantification and Association With Response to Immune Checkpoint Blockade in NonSmall-Cell Lung Cancer. JCO Precis Oncol 2019. doi: 10.1200/PO.19.00171

19. Chen DS, Mellman I. Elements of cancer immunity and the cancer-immune set point. Nature 2017;541:321-30.

20. Ott PA, Bang YJ, Piha-Paul SA, et al. T-Cell-Inflamed Gene-Expression Profile, Programmed Death Ligand 1 Expression, and Tumor Mutational Burden Predict Efficacy in Patients Treated With Pembrolizumab Across 20 Cancers: KEYNOTE-028. J Clin Oncol 2019;37:318-27.

21. van der Heijden EH, Casal RF, Trisolini R, et al. Guideline for the acquisition and preparation of conventional and endobronchial ultrasound-guided transbronchial needle aspiration specimens for the diagnosis and molecular testing of patients with known or suspected lung cancer. Respiration 2014;88:500-17.

22. Steinfort DP, Siva S, Leong TL, et al. Systematic
Endobronchial Ultrasound-guided Mediastinal Staging Versus Positron Emission Tomography for Comprehensive Mediastinal Staging in NSCLC Before Radical Radiotherapy of Non-small Cell Lung Cancer: A Pilot Study. Medicine (Baltimore) 2016;95:e2488.

23. Bonney A, Christie M, Beaty A, et al. The feasibility of molecular testing on cell blocks created from brush tip washings in the assessment of peripheral lung lesions. J Thorac Dis 2016;8:2551-5.

24. Steinfort DP, Bonney A, See K, et al. Sequential multimodality bronchoscopic investigation of peripheral pulmonary lesions. Eur Respir J 2016;47:607-14.

25. Steinfort DP, Hew MJ, Irving LB. Bronchoscopic evaluation of the mediastinum using endobronchial ultrasound: a description of the first 216 cases carried out at an Australian tertiary hospital. Intern Med J 2011;41:815-24.

26. Steinfort DP, Khor YH, Manser RL, et al. Radial probe endobronchial ultrasound for the diagnosis of peripheral lung cancer: systematic review and meta-analysis. Eur Respir J 2011;37:902-10.

27. Steinfort DP, Russell PA, Tsui A, et al. Interobserver agreement in determining non-small cell lung cancer subtype in specimens acquired by EBUS-TBNA. Eur Respir J 2012;40:699-705.

28. Hendry S, Byrne DJ, Christie M, et al. Adequate tumour cellularity is essential for accurate PD-L1 immunohistochemistry assessment on cytology cell-block specimens. Cytopathology 2020;31:90-5.

29. Bonney A, Beaty A, See K, et al. Diagnostic Utility of Bronchial Brush-Tip Washings for the Immunohistochemical Assessment of Peripheral Lung Lesions. Acta Cytol 2016;60:74-8.

30. She S, Steinfort DP, Ing AJ, et al. Transbronchial Cryobiopsy in Interstitial Lung Disease: Safety of a Standardized Procedure. J Bronchology Interv Pulmonol 2020;27:36-41.

31. Arimura K, Kondo M, Nagashima Y, et al. Comparison of tumor cell numbers and 22C3 PD-L1 expression between cryobiopsy and transbronchial biopsy with endobronchial ultrasonography-guide sheath for lung cancer. Respir Res 2019;20:185.

32. Steinfort DP, D'Agostino RD, Vrjlic I, et al. CTFluoroscopic Guidance for Performance of Targeted Transbronchial Cryobiopsy: A Preliminary Report. Respiration 2018;96:472-9.

33. Leong TL, Loveland PM, Gorelik A, et al. Preoperative Staging by EBUS in cN0/N1 Lung Cancer: Systematic 
Review and Meta-Analysis. J Bronchology Interv Pulmonol 2019;26:155-65.

34. Leong TL, Christie M, Kranz S, et al. Evaluating the Genomic Yield of a Single Endobronchial Ultrasoundguided Transbronchial Needle Aspiration in Lung Cancer: Meeting the Challenge of Doing More With Less. Clin Lung Cancer 2017;18:e467-72.

35. Leong TL, Gayevskiy V, Steinfort DP, et al. Deep multiregion whole-genome sequencing reveals heterogeneity and gene-by-environment interactions in treatment-naive, metastatic lung cancer. Oncogene 2019;38:1661-75.

36. Murthy V, Katzman DP, Tsay JJ, et al. Tumor-draining lymph nodes demonstrate a suppressive immunophenotype in patients with non-small cell lung cancer assessed by endobronchial ultrasound-guided transbronchial needle aspiration: A pilot study. Lung Cancer 2019;137:94-9.

37. Clave S, Pijuan L, Casadevall D, et al. CD274 (PDL1) and JAK2 genomic amplifications in pulmonary squamouscell and adenocarcinoma patients. Histopathology 2018;72:259-69.

38. Erber R, Stohr R, Herlein S, et al. Comparison of PDL1 mRNA Expression Measured with the CheckPoint Typer(R) Assay with PD-L1 Protein Expression Assessed with Immunohistochemistry in Non-small Cell Lung Cancer. Anticancer Res 2017;37:6771-8.

39. Kim GJ, Lee JH, Park WJ, et al. The Prognostic Value of Measuring PD-L1 mRNA Expression Levels in Surgically Resected Non-Small Cell Lung Cancer. Ann Clin Lab Sci 2019;49:317-23.

40. Vannitamby A, Hendry S, Makadia T, et al. A Novel Approach to Detect Programed Death Ligand 1 (PDL1) Status and Multiple Tumor Mutations Using a Single Non-Small-Cell Lung Cancer (NSCLC) Bronchoscopy Specimen. J Mol Diagn 2019;21:186-97.

41. Sacher AG, Paweletz C, Dahlberg SE, et al. Prospective Validation of Rapid Plasma Genotyping for the Detection of EGFR and KRAS Mutations in Advanced Lung Cancer. JAMA Oncol 2016;2:1014-22.

42. Yung TKF, Chan KCA, Mok TSK, et al. Single-Molecule Detection of Epidermal Growth Factor Receptor Mutations in Plasma by Microfluidics Digital PCR in Non-Small Cell Lung Cancer Patients. Clinical Cancer Research 2009;15:2076-84.

43. Vannitamby A, Hendry S, Irving L, et al. Novel multiplex droplet digital PCR assay for scoring PD-L1 in nonsmall cell lung cancer biopsy specimens. Lung Cancer 2019;134:233-7.

44. Kowanetz M, Zou W, Gettinger SN, et al. Differential regulation of PD-L1 expression by immune and tumor cells in NSCLC and the response to treatment with atezolizumab (anti-PD-L1). Proc Natl Acad Sci U S A 2018;115:E10119-26.

45. Garcia-Diaz A, Shin DS, Moreno BH, et al. Interferon Receptor Signaling Pathways Regulating PD-L1 and PDL2 Expression. Cell Rep 2017;19:1189-201.

46. Ribas A RC, Hodi S, Wolchok J, et al. Association of response to programmed death receptor 1 (PD-1) blockade with pembrolizumab (MK-3475) with an interferoninflammatory immune gene signature. J Clin Oncol 2015;33:3001.

47. Herbst RS, Soria JC, Kowanetz M, et al. Predictive correlates of response to the anti-PD-L1 antibody MPDL3280A in cancer patients. Nature 2014;515:563-7.

48. Janes MR, Zhang J, Li LS, et al. Targeting KRAS Mutant Cancers with a Covalent G12C-Specific Inhibitor. Cell 2018;172:578-89.e17.

49. Jiang X, Zhou J, Giobbie-Hurder A, et al. The activation of MAPK in melanoma cells resistant to BRAF inhibition promotes PD-L1 expression that is reversible by MEK and PI3K inhibition. Clin Cancer Res 2013;19:598-609.

50. Coelho MA, de Carne Trecesson S, Rana S, et al. Oncogenic RAS Signaling Promotes Tumor Immunoresistance by Stabilizing PD-L1 mRNA. Immunity 2017;47:1083-99.e6.

51. Passiglia F, Cappuzzo F, Alabiso O, et al. Efficacy of nivolumab in pre-treated non-small-cell lung cancer patients harbouring KRAS mutations. Br J Cancer 2019;120:57-62.

52. Jeanson A, Tomasini P, Souquet-Bressand M, et al. Efficacy of Immune Checkpoint Inhibitors in KRAS-Mutant NonSmall Cell Lung Cancer (NSCLC). J Thorac Oncol 2019;14:1095-101.

53. Lee CK, Man J, Lord S, et al. Clinical and Molecular Characteristics Associated With Survival Among Patients Treated With Checkpoint Inhibitors for Advanced NonSmall Cell Lung Carcinoma: A Systematic Review and Meta-analysis. JAMA Oncol 2018;4:210-6.

54. Kim JH, Kim HS, Kim BJ. Prognostic value of KRAS mutation in advanced non-small-cell lung cancer treated with immune checkpoint inhibitors: A meta-analysis and review. Oncotarget 2017;8:48248-52.

55. Chen N, Fang W, Zhan J, et al. Upregulation of PDL1 by EGFR Activation Mediates the Immune Escape in EGFR-Driven NSCLC: Implication for Optional Immune Targeted Therapy for NSCLC Patients with EGFR Mutation. J Thorac Oncol 2015;10:910-23. 
56. Berghoff AS, Bellosillo B, Caux C, et al. Immune checkpoint inhibitor treatment in patients with oncogeneaddicted non-small cell lung cancer (NSCLC): summary of a multidisciplinary round-table discussion. ESMO Open 2019;4:e000498.

57. Evans M, O'Sullivan B, Hughes F, et al. The Clinicopathological and Molecular Associations of PD-L1 Expression in Non-small Cell Lung Cancer: Analysis of a Series of 10,005 Cases Tested with the 22C3 Assay. Pathol Oncol Res 2020;26:79-89.

58. Ota K, Azuma K, Kawahara A, et al. Induction of PDL1 Expression by the EML4-ALK Oncoprotein and Downstream Signaling Pathways in Non-Small Cell Lung Cancer. Clin Cancer Res 2015;21:4014-21.

59. Lim WT, Zhang WH, Miller CR, et al. PTEN and phosphorylated AKT expression and prognosis in earlyand late-stage non-small cell lung cancer. Oncol Rep 2007;17:853-7.

60. Tang JM, He QY, Guo RX, et al. Phosphorylated Akt

Cite this article as: Bozinovski S, Vannitamby A, Rangamuwa K, Aujla S, Wang H, Aloe C, Irving L, Leong TT, Steinfort DP. Integrating endobronchial ultrasound bronchoscopy with molecular testing of immunotherapy biomarkers in non-small cell lung cancer. Transl Lung Cancer Res 2021;10(6):27792787. doi: 10.21037/tlcr-20-781 overexpression and loss of PTEN expression in non-small cell lung cancer confers poor prognosis. Lung Cancer 2006;51:181-91.

61. Marsit CJ, Zheng S, Aldape K, et al. PTEN expression in non-small-cell lung cancer: evaluating its relation to tumor characteristics, allelic loss, and epigenetic alteration. Hum Pathol 2005;36:768-76.

62. Drilon A, Rekhtman N, Ladanyi M, et al. Squamous-cell carcinomas of the lung: emerging biology, controversies, and the promise of targeted therapy. Lancet Oncol 2012;13:e418-26.

63. Soria JC, Lee HY, Lee JI, et al. Lack of PTEN expression in non-small cell lung cancer could be related to promoter methylation. Clin Cancer Res 2002;8:1178-84.

64. Lastwika KJ, Wilson W 3rd, Li QK, et al. Control of PD-L1 Expression by Oncogenic Activation of the AKTmTOR Pathway in Non-Small Cell Lung Cancer. Cancer Res 2016;76:227-38. 